\title{
Loss of Dopamine Transporters in Methamphetamine Abusers Recovers with Protracted Abstinence
}

\author{
Nora D. Volkow, ${ }^{1,3}$ Linda Chang,, ${ }^{1,3}$ Gene-Jack Wang, ${ }^{1}$ Joanna S. Fowler, ${ }^{2}$ Dinko Franceschi, ${ }^{1}$ Mark Sedler, ${ }^{3}$ \\ Samuel J. Gatley, ${ }^{1}$ Eric Miller, ${ }^{4}$ Robert Hitzemann, ${ }^{3}$ Yu-Shin Ding, ${ }^{1}$ and Jean Logan ${ }^{1}$ \\ ${ }^{1}$ Medical and ${ }^{2}$ Chemistry Departments, Brookhaven National Laboratory, Upton, New York 11973, ${ }^{3}$ Department of \\ Psychiatry, State University of New York at Stony Brook, Stony Brook, New York 11794, and ${ }^{4}$ Department of Psychiatry, \\ Harbor-University of California, Los Angeles, Torrance, California 90502
}

\begin{abstract}
Methamphetamine is a popular drug of abuse that is neurotoxic to dopamine (DA) terminals when administered to laboratory animals. Studies in methamphetamine abusers have also documented significant loss of DA transporters (used as markers of the DA terminal) that are associated with slower motor function and decreased memory. The extent to which the loss of DA transporters predisposes methamphetamine abusers to neurodegenerative disorders such as Parkinsonism is unclear and may depend in part on the degree of recovery. Here we assessed the effects of protracted abstinence on the loss of DA transporters in striatum, in methamphetamine abusers using positron emission tomography and $\left[{ }^{11} \mathrm{C}\right] d$-threo-methylphenidate (DA transporter radioligand). Brain DA transporters in five methamphetamine abusers evaluated during short abstinence $(<6$ months) and then retested during protracted abstinence (12-17 months) showed significant increases with protracted abstinence (caudate, $+19 \%$; putamen, $+16 \%$ ). Although performance in
\end{abstract}

The rapidly escalating abuse of methamphetamine (METH) places a sense of urgency on understanding its effects on the human brain and its medical consequences. METH is a particularly problematic drug in that not only is it highly addictive but its administration to laboratory animals results in damage to dopamine (DA) terminals (Seiden and Sabol, 1996). Studies in humans have also documented a significant loss of DA transporters (DAT), which have been used as markers of DA terminals, in the brains of METH abusers (Wilson et al., 1996; McCann et al., 1998; Volkow et al., 2001). Although the DAT loss reported in METH abusers $(24-30 \%)$ is smaller than that reported in Parkinson's disease (36 and 71\%) (Frost et al., 1993; Innis et al., 1993), it is nonetheless associated with reduced motor speed and impaired verbal learning (Volkow et al., 2001). Because significant reductions in DAT occur both with age $(6-7 \%$ per decade

Received Aug. 8, 2001; revised Sept. 13, 2001; accepted Sept. 19, 2001.

This research was performed at Brookhaven National Laboratory and was supported by United States Department of Energy Office of Biological and Environmental Research Grant DE-AC02-98CH10886, National Institute on Drug Abuse Grants DA06891, DA7092-01, and DA00280, General Clinical Research Center (GCRC) at Harbor-University of California, Los Angeles Medical Center National Institutes of Health Grant MO1RR 00425, GCRC at University Hospital Stony Brook National Institutes of Health Grant MO1RR 10710, and the Office of National Drug Control Policy. We thank D. Schlyer and R. Carciello for Cyclotron operations, D. Warner for PET operations, C. Wong for data management, R. Ferrieri, C. Shea, R. MacGregor, V. Garza, and P. King for radiotracer preparation and analysis, and N. Pappas, N. Netusil, and Pauline Carter for patient care.

Correspondence should be addressed to Nora D. Volkow, Medical Department, Brookhaven National Laboratory, Upton, NY 11973. E-mail: volkow@bnl.gov.

Copyright (C) 2001 Society for Neuroscience 0270-6474/01/219414-05\$15.00/0 some of the tests for which we observed an association with DA transporters showed some improvement, this effect was not significant. The DA transporter increases with abstinence could indicate that methamphetamine-induced DA transporter loss reflects temporary adaptive changes (i.e., downregulation), that the loss reflects DA terminal damage but that terminals can recover, or that remaining viable terminals increase synaptic arborization. Because neuropsychological tests did not improve to the same extent, this suggests that the increase of the DA transporters was not sufficient for complete function recovery. These findings have treatment implications because they suggest that protracted abstinence may reverse some of methamphetamine-induced alterations in brain DA terminals.

Key words: methamphetamine; dopamine transporters; imaging; positron emission tomography; addiction; detoxification; neurotoxicity; dopamine terminal for the 20-80 year age span) (Volkow et al., 1996) and with METH abuse (equivalent to that seen over 40 years of aging), a concern arises as to whether the DAT losses in the METH abusers will place them at risk for Parkinsonism as they age. The extent to which this is likely to occur will depend in part on the reversibility of the changes induced by METH.

Although previous studies proposed that METH-induced DAT losses reflected irreversible terminal degeneration (Ricaurte and McCann, 1992), recent studies in rodents (Friedman et al., 1998; Cass and Manning 1999) and in nonhuman primates (Melega et al., 1997; Harvey et al., 2000) have revealed significant recovery with protracted abstinence. This suggests that either METHinduced damage to the DA terminals recovers or that the DAT losses reflect adaptive changes rather than neurotoxicity. The extent to which a similar recovery may occur in METH abusers has not been investigated. Here we investigate whether the DAT losses in METH abusers recover with protracted abstinence.

For this purpose, we used positron emission tomography (PET) and the DAT radioligand $\left[{ }^{11} \mathrm{C}\right]$ d-threo-methylphenidate (Volkow et al., 1995) to measure DAT availability in METH abusers. The initial group, which consisted of $12 \mathrm{METH}$ abusers, was tested within 6 months of the last METH administration (Volkow et al., 2001). They were followed and retested at least 9 months later if they remained drug free. Only five METH abusers who were able to stay drug-free were retested at least 9 months later (protracted abstinence). Here we report on the results for the repeated studies in these subjects and on those of five additional METH 
abusers who were tested only once after having been abstinent for at least 9 months. The DAT measures were compared with those in controls and with those we reported previously in METH abusers tested within 6 months of abstinence (Volkow et al., 2001).

\section{MATERIALS AND METHODS}

Subjects. Five METH abusers (three females, two males; $29 \pm 3$ years of age) were evaluated twice: during early (mean $\pm \mathrm{SD}, 3 \pm 1.6$ months of abstinence) and then at least 9 months later during protracted abstinence (mean $\pm \mathrm{SD}, 14 \pm 2$ months). These five METH abusers were the ones from an original sample of 12 METH abusers (Volkow et al., 2001) who were able to remain drug free for at least 9 months since the first PET scan evaluation. In addition, we also evaluated five METH abusers (four females, one male; $35 \pm 3$ years of age) who were tested only once after having been detoxified for at least 9 months $(17 \pm 10$ months of abstinence). METH abusers fulfilled Diagnostic and Statistical Manual of Mental Disorders IV criteria for METH dependence (average METH use of at least $0.25 \mathrm{gm} / \mathrm{d}$, at least $5 \mathrm{~d}$ per week for at least 2 years, at least 2 weeks of METH abstinence). Subjects were excluded if seropositive for human immunodeficiency virus, comorbid neuropsychiatric disorder, medical illness, or current or past history of drug dependence other than METH and nicotine. Details for screening have been published previously (Volkow et al., 2001). METH abusers were enrolled in a California drug-court monitoring rehabilitation program and were evaluated weekly or biweekly with drug screens to ensure lack of drug use during the abstinence period. Controls were 11 healthy non-drug-abusing subjects (seven females, four males; $31 \pm 7$ years of age) who were screened for lack of a past or present history of drug abuse (except for nicotine or caffeine). Prescan urine tests ensured absence of psychoactive drug use in all subjects. The protocol was approved by the Institutional Review Boards at Brookhaven National Laboratory, State University of New York at Stony Brook, and Harbor-University of California, Los Angeles Medical Center. All subjects gave written informed consent after the experimental procedure was explained and after they had read the consent form.

Scanning. PET scans were performed using a CTI 931 Siemens scanner (spatial resolution, $6 \times 6 \times 6.5 \mathrm{~mm}$ full-width half-maximum). Dynamic scans were started after intravenous injection of $4-8 \mathrm{mCi}$ of $\left[{ }^{11} \mathrm{C}\right] d$ threo-methylphenidate (specific activity $>0.4 \mathrm{Ci} / \mu \mathrm{mol}$ at time of injection) for a total of $84 \mathrm{~min}$ as described previously (Volkow et al., 1995).

Neuropsychological evaluation. In the METH abusers, we assessed performance in neuropsychological tasks, which we showed to be correlated with DAT levels (Volkow et al., 2001). The tests were performed within 2 weeks of the PET studies and included tests for the following: (1) motor function: gross motor function (timed gait, subject walks as fast as possible in a straight line) and fine motor coordination (grooved pegboard, subject inserts pegs in small holes angled in different directions as quickly as possible); and (2) memory function: Rey auditory verbal learning test (subject has to learn and recall lists of unrelated words immediately, after a time delay, and after a distractor).

Image analysis and modeling. Regions in striatum (caudate and putamen) were obtained from three sequential planes and in the cerebellum (CB) from two sequential planes and were drawn on the averaged emission scans (activity between 10 and $84 \mathrm{~min}$ ). Regions in striatum are selected in multiple planes to increase the reproducibility of the measures (Bendriem et al., 1991). The regions were then projected to the dynamic emission scans to obtain tissue time activity curves. These tissue time activity curves along with the time activity curves for unchanged tracer in plasma were used to calculate the transfer constant of $\left[{ }^{11} \mathrm{C}\right]$ d-threomethylphenidate from plasma to brain $\left(K_{1}\right)$ and the distribution volumes (DV), which correspond to the equilibrium measurement of the ratio of tissue concentration to plasma concentration, in striatum and CB using a graphical analysis technique for radiotracers with reversible kinetics (Logan et al., 1990). The ratio of DV in striatum to that in cerebellum, which corresponds to $\left(B_{\max } / K_{\mathrm{d}}\right)+1$ and is insensitive to changes in cerebral blood flow (Logan et al., 1994), was used as measure of DAT availability.

Statistical analysis. Differences in $K_{1}$ and $B_{\max } / K_{\mathrm{d}}$ between the measures obtained in the METH abusers tested during short and protracted abstinence were tested with paired $t$ tests (two tailed). Pearson product correlations were used to assess the relationship between the changes in $B_{\max } / K_{\mathrm{d}}$ and the lapse in days between the first and the second evaluation and the doses and the years of METH use. Differences in neuropsycho-

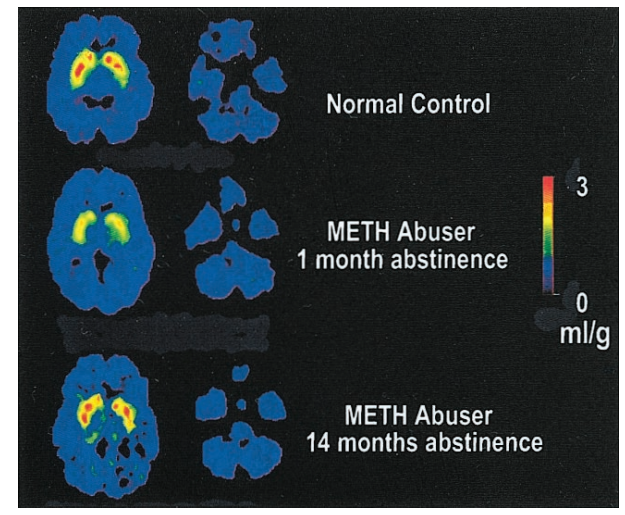

Figure 1. Brain images of the distribution volume of $\left[{ }^{11} \mathrm{C}\right]$ d-threomethylphenidate in a control and a METH abuser. Images shown were obtained at the level of the striatum (images to the left) and the cerebellum (images to the right), and they are from a normal control and a METH abuser evaluated twice, during short and protracted abstinence. Notice the significant increases in binding in striatum in the METH abuser with protracted abstinence.

logical performance between the first and second evaluation were tested with paired $t$ tests. Pearson's product correlations were used to assess the relationship between the changes in DAT and the changes in neuropsychological scores between the first and the second evaluation.

Differences in $K_{1}$ and $B_{\max } / K_{\mathrm{d}}$ between the controls and the METH abusers evaluated during protracted abstinence (five METH abusers evaluated only once during protracted abstinence and the data obtained during the second evaluation for the five METH abusers tested twice) and those in the original sample of $12 \mathrm{METH}$ abusers tested during short abstinence (seven females, five males; $30 \pm 6$ years of age; $64 \pm 40 \mathrm{~d}$ of abstinence) (Volkow et al., 2001) were tested with factorial ANOVA. Post hoc $t$ tests were done to assess the differences that were significant. Differences between the METH abusers who might have relapsed within 9 months of the first PET evaluation (seven subjects who dropped out from the detoxification program) and those that did not (five subjects) were tested with factorial ANOVA.

\section{RESULTS}

\section{DAT in the five METH abusers tested twice during short and protracted abstinence}

In the METH abusers evaluated twice, there were no differences between short and protracted abstinence for $K_{1}$ in cerebellum $(0.45 \pm 0.10$ vs $0.47 \pm 0.11)$ or in caudate $(0.55 \pm 0.08$ vs $0.55 \pm$ $0.08)$ or putamen $(0.63 \pm 0.10$ vs $0.63 \pm 0.09)$. However, binding of $\left[{ }^{11} \mathrm{C}\right] d$-threo-methylphenidate in striatum, but not in cerebellum, was increased between the short and the protracted abstinence (Fig. 1). DAT availability $\left(B_{\max } / K_{\mathrm{d}}\right)$ was significantly increased from short to protracted abstinence in caudate $(+19 \%$; $p<0.003)$ and putamen $(+16 \% ; p<0.05)$ (Fig. 2$)$. The magnitude of the changes in DAT in putamen were negatively correlated with the reported doses of METH used $(r=0.88$; $\mathrm{df}=4$; $p<0.05)$, and there was a trend for a negative correlation with the years of METH use $(r=0.83$; df $=4 ; p<0.08)($ Fig. $3 A)$. The later correlations were not significant for the changes in caudate. The correlation between the days between evaluations and the magnitude of the increases in DAT was significant for the averaged changes in caudate and putamen $(r=0.92$; $\mathrm{df}=4 ; p<0.05)$ (Fig. 3B).

\section{Neuropsychological performance in METH abusers tested twice during short and protracted abstinence}

Performance in the neuropsychological tests for which we hypothesized a priori an association with DAT measures showed a nonsignificant trend of improvement for timed gait $(10.0 \pm 1$ vs 

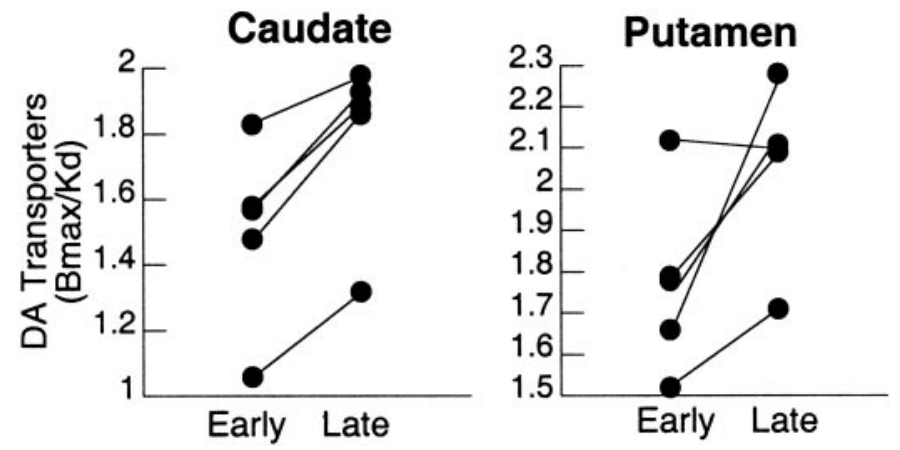

Figure 2. Individual measures for DAT availability in the five METH abusers tested twice during short and protracted abstinence. Measures of DAT availability $\left(B_{\max } / K_{\mathrm{d}}\right)$ were significantly increased with protracted abstinence in caudate $(p<0.003)$ and putamen $(p<0.05)$. Repeated-measures ANOVA.

$8.6 \pm 2 ; p<0.13)$ and the delayed recall $(11.6 \pm 3$ vs $12.8 \pm 2 ; p<$ $0.10)$ but no changes in the pegboard or the immediate or interference recall between the first and second evaluations. Although not significant, there was also a trend for an association between the changes in DAT between the first and second evaluation and the changes in the scores for timed gait $(r=0.78 ; p<0.12)$ and the delayed recall $(r=0.71 ; p<0.18)$.

\section{Comparison between controls and METH abusers tested during short or protracted abstinence}

Comparison of DAT availability $\left(B_{\max } / K_{\mathrm{d}}\right)$ between the METH groups tested during short versus those tested during protracted abstinence (these two groups combined the data from METH tested only once and the data from the five METH abusers tested twice) versus controls showed a significant group effect in caudate $(F=7.9 ; \mathrm{df}=2,30 ; p<0.002)$ and putamen $(F=5.5 ; \mathrm{df}=2,30$; $p<0.01)$. Post hoc $t$ tests showed that the $B_{\max } / K_{\mathrm{d}}$ measures in METH abusers tested during short abstinence were significantly lower than those in controls (caudate, 26\%; putamen, 21\%; $p<$ 0.001), whereas those in the METH abusers tested during protracted abstinence, although slightly lower, did not differ significantly from those in controls (caudate, 5\%; putamen, 9\%; NS) (Table 1). Differences were also significant between the METH abusers evaluated during short and those evaluated during protracted abstinence in caudate (26\% difference; $p<0.01)$, and there was a trend in putamen $(15 \%$ difference; $p<0.10)$ (Table
Table 1. Measures of $K_{1}$ and DAT availability in controls and methamphetamine abusers evaluated during short or protracted abstinence

\begin{tabular}{clll} 
& $\begin{array}{l}\text { Controls } \\
(n=11)\end{array}$ & $\begin{array}{l}\text { METH abusers } \\
(\text { short abstinence) } \\
(n=12)\end{array}$ & $\begin{array}{l}\text { METH abusers } \\
\left(\begin{array}{l}\text { protracted abstinence }) \\
(n=10)\end{array}\right.\end{array}$ \\
\hline$K_{1}$ & & & \\
Cerebellum & $0.42 \pm 0.11$ & $0.48 \pm 0.17$ & $0.46 \pm 0.11$ \\
Caudate & $0.58 \pm 0.12$ & $0.63 \pm 0.18$ & $0.55 \pm 0.07$ \\
$\begin{array}{c}\text { Putamen } \\
B_{\text {max }} / K_{\mathrm{d}}\end{array}$ & $0.64 \pm 0.14$ & $0.73 \pm 0.24$ & $0.62 \pm 0.08$ \\
Caudate & $1.76 \pm 0.27$ & $1.32 \pm 0.28^{*}$ & $1.67 \pm 0.29^{* *}$ \\
Putamen & $1.97 \pm 0.21$ & $1.56 \pm 0.30^{*}$ & $1.80 \pm 0.35$
\end{tabular}

The $K_{1}$ (transfer constant of $\left[{ }^{11} \mathrm{C}\right] d$-threo-methylphenidate from plasma to brain) and $B_{\max } / K_{\mathrm{d}}$ (measure of DAT availability) were measured in 11 controls, $12 \mathrm{METH}$ abusers evaluated during short abstinence ( $64 \pm 40 \mathrm{~d}$ of abstinence), and $10 \mathrm{METH}$ abusers evaluated during protracted abstinence ( $15 \pm 7$ months of abstinence). Note that the data for the five METH abusers tested twice was included in this group analysis: the data obtained during the first evaluation as part of the group tested during short abstinence and the data obtained during the second evaluation as part of the group tested during protracted abstinence. Factorial ANOVA showed that the group differences were significant for $B_{\text {max }} / K_{\mathrm{d}}$ in caudate $(p<0.002)$ and putamen $(p<0.01)$. Post hoc $t$ tests; ${ }^{*} p<0.003$, different from controls; ${ }^{* *} p<0.01$, different from short abstinence.

1). There were no differences between groups on the $K_{1}$ measures (Table 1).

Comparison between METH abusers who dropped out of the detoxification program and those that did not

Comparison of striatal DAT availability showed that subjects who dropped out of the detoxification program (most likely relapsed) had significantly lower DAT levels during the short abstinence period than those who completed the detoxification in putamen $(1.40 \pm 0.3$ vs $1.74 \pm 0.2 ; \mathrm{df}=10 ; p<0.03)$ and had a trend for lower values in caudate $(1.20 \pm 0.2$ vs $1.48 \pm 0.3$; df $=10 ; p<$ 0.07). The METH abusers who dropped out performed worse in the neuropsychological tests than those that did not, and these were significant for the pegboard (92 \pm 19 vs $67 \pm 9$; $\mathrm{df}=10 ; p<$ $0.05)$ and the delayed recall ( $8 \pm 2$ vs $12 \pm 3$; df $=10 ; p<0.05)$.

\section{DISCUSSION}

\section{Recovery of DAT with protracted METH abstinence}

This study documents significant recovery of DAT with protracted abstinence in METH abusers who were able to stay drug free for at least 9 months after the initial evaluation. Moreover,
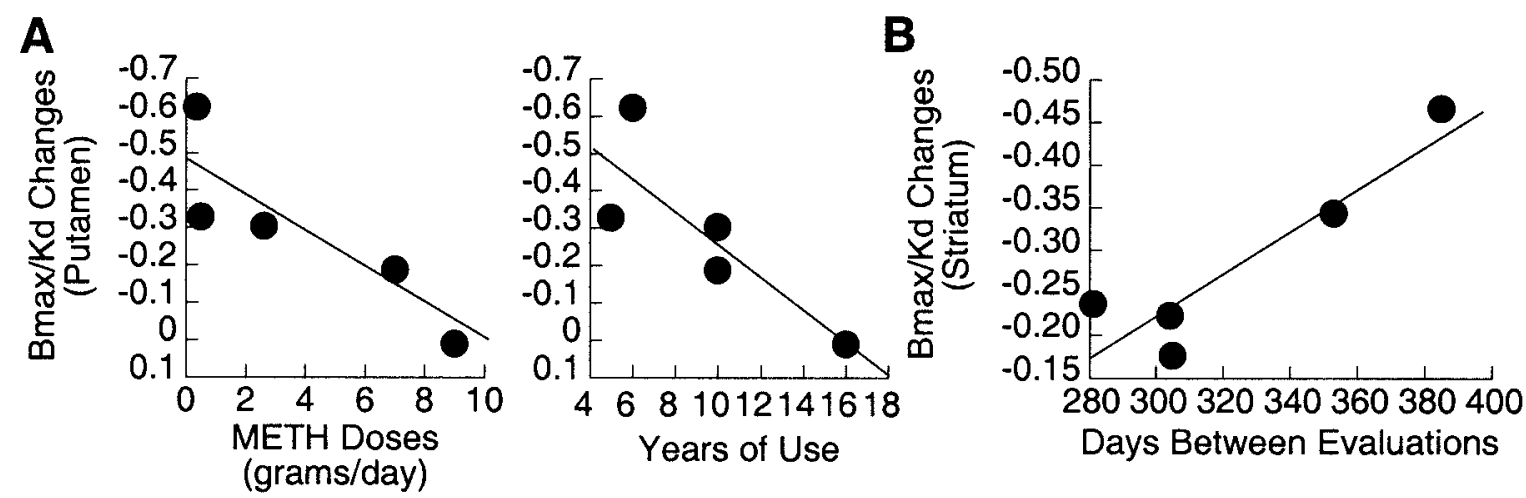

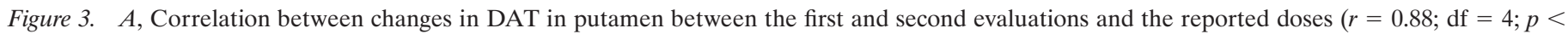

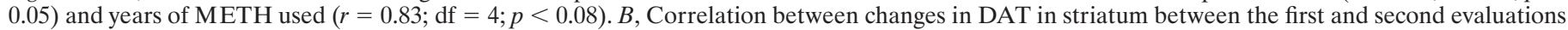
and the days elapsed between evaluations $(r=0.92$; df $=4 ; p<0.05)$. 
the longer the period between the first and the second evaluation the larger the increases in DAT, suggesting that DAT recovery is in part a function of the length of the abstinence period.

The increases in DAT with protracted abstinence are consistent with recent studies in laboratory animals, which have documented significant recovery from METH-induced DA terminal changes. These studies have shown results that vary from complete recovery to persistent long-lasting changes after METH administration. Complete recovery was reported in a study done in rats, which showed marked reduction in baseline DA levels and in amphetamine-induced increases in DA release 1 week after METH that recovered to normal values 12 months after treatment (Cass and Manning, 1999). Significant, but not complete, recovery in DA function after METH administration was reported by another rodent study that showed marked DA depletions 1 week after METH (30\% of controls) that recovered 7 months later ( $80 \%$ of controls) (Friedman et al., 1998). Similarly, imaging studies in nonhuman primates have shown significant recovery of 6-[18F]fluoro-L-dihydroxyphenylalanine and dopamine concentrations at 3-4 months (Melega et al., 1997) and in DAT at 12-15 months after METH treatment (Harvey et al., 2000). In the latter study, the histological analyses of the brains did not reveal evidence of axonal regeneration, as would have been expected if the DAT recovery had been attributable to regeneration of previously damaged terminals (Harvey et al., 2000). However, other studies have reported marked striatal DA reductions in rhesus monkeys even 4 years after METH treatment (Woolverton et al., 1989). These discrepancies are likely to reflect the fact that the extent of recovery may vary across species and age of the animals (Miller et al., 2000) and that it is dependent on the pattern and the amount of METH administered.

\section{Rate of DAT recovery is associated with severity of abuse and days of abstinence}

In the METH abusers who were tested twice, the degree of recovery in DAT with protracted abstinence was negatively correlated with the doses and year of METH used, which indicates that severity may ultimately limit the amount and speed of recovery. Similar findings have been reported in laboratory animals for whom the rate of recovery was also shown to be correlated with the doses of METH that were administered to the animals (Bakhit and Gibb, 1981). The fact that the correlation was significant for putamen but did not reach significance for caudate is likely to reflect the low power afforded by the small sample of subjects in whom we were able to obtain follow-up PET scans. The magnitude of the DAT recovery with abstinence was also positively correlated with the time between evaluations. Similar findings are reported in the animal literature in which studies have shown increasingly greater recovery as a function of the days since METH pretreatment (Melega et al., 1997; Friedman et al., 1998).

\section{Comparisons between controls, METH abusers tested during short abstinence, and METH abusers tested during protracted abstinence}

Although DAT in METH abusers tested during protracted abstinence did not differ from controls, they were slightly lower. Moreover, failure to achieve a parallel recovery of neuropsychological function with METH abstinence suggests that the lower DAT levels in METH abusers could reflect an incomplete recovery. Similarly, primate studies that reported significant recovery with METH detoxification also described some remaining persistent deficits (Harvey et al., 2000).
Comparisons of DAT between the METH abusers group tested during short abstinence and the group tested during protracted abstinence showed significantly higher DAT in METH abusers tested during protracted abstinence. This again corroborates a significant recovery of DAT with protracted abstinence. The fact that there were no differences in $K_{1}$ (measure of tracer transport from plasma to brain) between the groups indicates that the changes observed in $B_{\max } / K_{\mathrm{d}}$ in the METH abusers tested during short but not during protracted abstinence reflect changes in DAT availability and not nonspecific effects of tracer delivery into the brain.

\section{Interpretation of DAT losses with METH and their recovery with protracted abstinence}

The losses in DAT induced by METH had been attributed to irreversible neurodegeneration of DA terminals (Ricaurte and McCann, 1992). However, recent studies documenting significant recovery of DAT with abstinence have started to question this interpretation. Also, a postmortem study done in METH abusers that showed reductions in DAT but not in vesicular monoamine transporters (VMAT) interpreted the persistence of VMAT, which is a more stable markers of the DA terminals than DAT (Wilson and Kish, 1996), as an indication of preservation of the DA terminal (Wilson and Kish, 1996). However, inferences from VMAT measures are limited by the fact that VMAT is also expressed in cells other than DA neurons (Schutz et al., 1998).

The DAT recovery with protracted abstinence could reflect either that METH-induced damage to the DA terminals recovers or that the DAT losses reflect adaptive changes (DAT downregulation or internalization). Evidence that METH can decrease protein expression in DA terminals was shown by a study done in rodents that documented METH-induced decreases in mRNA for VMAT and tyrosine hydroxylase (Zhang and Angulo, 1996). METH-induced decreases in DAT could also result from disruption of the process regulating DAT trafficking and internalization (Melikian and Buckley, 1999). Although internalization of DAT by METH has not been documented, it has been shown for amphetamine (Saunders et al., 2000). However, the time course of the DAT recovery (recovery of DAT after METH discontinuation in both laboratory animals and humans takes longer than 6-9 months) is longer than would be expected if the DAT losses were attributable to DAT downregulation or internalization (Iwata et al., 1999; Bachvarov et al., 2001).

An alternative explanation is that the DAT losses reflect DA terminal degeneration and that the DAT increases with protracted abstinence could result from regeneration of the DA nerve terminals or branching of the remaining terminals as an attempt to increase synaptic density in striatum. It is also possible that viable DA terminals overexpress DAT. However, the latter is unlikely because it would result in a decrease in extracellular DA concentration. Although studies have reported DAT upregulation after chronic stimulant administration (cocaine), this was shown shortly after drug discontinuation, and values returned to baseline with detoxification (Malison et al., 1998).

\section{Neuropsychological function did not recover with protracted abstinence}

Although DAT recovered significantly with protracted abstinence, neuropsychological function only showed a trend of improvement for some tests. Similarly, the relationship between the changes in DAT with abstinence and the changes in neuropsychological performance did not reach significance. Because we 
observed previously a correlation between performance on these tests and DAT levels, this suggests that, although there is recovery of DAT levels, there is not a parallel improvement in function. This could occur if the recovery reflects increased arborization of viable terminals that may still be insufficient to compensate for DA terminal loss or if the increases reflect upregulation of DAT in viable terminals. It is also possible that neuropsychological function requires other systems that may have been affected by METH for which recovery may be slower or not present. However, failure to see an association could reflect the poor power afforded by the small sample of subjects who were able to stay drug free. Prospective studies in larger samples of METH abusers are required to assess whether recovery of DAT with protracted abstinence is associated with recovery of neuropsychological function.

\section{Differences between METH abusers who dropped out of the detoxification program and those that did not}

At the time of the first evaluation, the METH abusers who were able to stay drug free had higher levels of DAT than the subjects who dropped out of the detoxification program. Thus, it is possible that in part their success to remain drug free was attributable to the fact that, to start with, they had less DA terminal damage than those subjects that were unsuccessful. It is also possible that the degree of DAT recovery is affected by the initial level of impairment and that the recovery in DAT contributed to the ability of the METH abusers to stay drug free. Studies comparing the rate of DAT recovery between METH abusers who are able to stay drug free and those who are not are needed to determine the generalizability of DAT recovery in METH abusers and its prognostic implications.

\section{Summary}

The results from this study provide evidence that the DAT losses in METH abusers recover significantly with protracted abstinence. The findings from these studies have implications in the treatment of METH abusers because they suggest that protracted abstinence and proper rehabilitation may reverse some of the METH-induced alterations in brain DA terminals.

\section{REFERENCES}

Bachvarov DR, Houle S, Bachvarova M, Bouthillier J, Adam A, Marceau F (2001) Bradykinin B(2) receptor endocytosis, recycling, and downregulation assessed using green fluorescent protein conjugates. J Pharmacol Exp Ther 297:19-26.

Bakhit C, Gibb JW (1981) Methamphetamine-induced depression of tryptophan hydroxylase: recovery following acute treatment. Eur J Pharmacol 76:229-233.

Bendriem B, Dewey SL, Schlyer DJ, Wolf AP, Volkow ND (1991) Quantitation of the human basal ganglia with positron emission tomography: a phantom study of the effect of contrast and axial positioning. IEEE Trans Med Imaging 10:216-222.

Cass WA, Manning MW (1999) Recovery of presynaptic dopaminergic functioning in rats treated with neurotoxic doses of methamphetamine. J Neurosci 19:7653-7660.

Friedman SD, Castañeda E, Hodge GK (1998) Long-term monoamine depletion, differential recovery, and subtle behavioral impairment following methamphetamine-induced neurotoxicity. Pharmacol Biochem Behav 61:35-44.

Frost JJ, Rosier AJ, Reich SG, Smith JS, Ehlers MD, Snyder SH, Ravert HT, Dannals RF (1993) Positron emission tomographic imaging of the dopamine transporter with ${ }^{11} \mathrm{C}$-W IN 35,428 reveals marked declines in mild Parkinson's disease. Ann Neurol 34:423-431.

Harvey DC, Lacan G, Tanious SP, Melega WP (2000) Recovery from methamphetamine induced long-term nigrostriatal dopaminergic deficits without substantia nigra cell loss. Brain Res 871:259-270.

Innis RB, Seibyl JP, Scanley BE, Laruelle M, Abi-Dargham A, Wallace E, Baldwin RM, Zea-Ponce Y, Zoghbi S, Wang S (1993) Single photon emission computed tomographic imaging demonstrates loss of striatal dopamine transporters in Parkinson disease. Proc Natl Acad Sci USA 90:11965-11969.

Iwata K, Ito K, Fukuzaki A, Inaki K, Haga T (1999) Dynamin and rab5 regulate GRK2-dependent internalization of dopamine D2 receptors. Eur J Biochem 263:596-602.

Logan J, Fowler JS, Volkow ND, Wolf AP, Dewey SL, Schlyer D, MacGregor RR, Hitzemann R, Bendriem B, Gatley SJ, Christman DR (1990) Graphical analysis of reversible binding from time activity measurements. J Cereb Blood Flow Metab 10:740-747.

Logan J, Volkow ND, Fowler JS, Wang G-J, Dewey SL, MacGregor R, Schlyer D, Gatley SJ, Pappas N, King P, Hitzemann R, Vitkun S (1994) Effects of blood flow on $\left[{ }^{11} \mathrm{C}\right]$ raclopride binding in the brain: model simulations and kinetic analysis of PET data. J Cereb Blood Flow Metab 14:995-1010.

Malison RT, Best SE, van Dyck CH, McCance EF, Wallace EA, Laruelle M, Baldwin RM, Seibyl JP, Price LH, Kosten TR, Innis RB (1998) Elevated striatal dopamine transporters during acute cocaine abstinence as measured by $\left[{ }^{123} \mathrm{I}\right]$ beta-CIT SPECT. Am J Psychiatry 155:832-834.

McCann UD, Wong DF, Yokoi F, Villemagne V, Dannals RF, Ricaurte GA (1998) Reduced striatal dopamine transporter density in abstinent methamphetamine and methcathinone users: evidence from positron emission tomography studies with $\left[{ }^{11} \mathrm{C}\right] \mathrm{W} I N-35,428$. J Neurosci 18:8417-8422.

Melega WP, Raleigh MJ, Stout DB, Lacan G, Huang SC, Phelps ME (1997) Recovery of striatal dopamine function after acute amphetamine- and methamphetamine-induced neurotoxicity in the vervet monkey. Brain Res 766:113-120.

Melikian HE, Buckley KM (1999) Membrane trafficking regulates the activity of the human dopamine transporter. J Neurosci 19:7699-7710.

Miller DB, O'Callaghan JP, Ali SF (2000) Age as a susceptibility factor in the striatal dopaminergic neurotoxicity observed in the mouse following substituted amphetamine exposure. Ann NY Acad Sci 914:194-207.

Ricaurte GA, McCann UD (1992) Neurotoxic amphetamine analogues: effects in monkeys and implications for humans. Ann NY Acad Sci 648:371-382.

Saunders C, Ferrer JV, Shi L, Chen J, Merrill G, Lamb ME, LeebLundberg LM, Carvelli L, Javitch JA, Galli A (2000) Amphetamineinduced loss of human dopamine transporter activity: an internalization-dependent and cocaine-sensitive mechanism. Proc Natl Acad Sci USA 97:6850-6855.

Schutz B, Schafer MK, Eiden LE, Weihe E (1998) Vesicular amine transporter expression and isoform selection in developing brain, peripheral nervous system and gut. Brain Res Dev Brain Res 106:181-204.

Seiden LS, Sabol KE (1996) Methamphetamine and methylenedioxymethamphetamine neurotoxicity: possible mechanisms of cell destruction. NIDA Res Monogr 163:251-276.

Volkow ND, Ding Y-S, Fowler JS, Wang G-J, Logan J, Gatley SJ, Schlyer DJ, Pappas N (1995) A new PET ligand for the dopamine transporter: Studies in the human brain. J Nucl Med 36:2162-2168.

Volkow ND, Ding Y-S, Fowler JS, Wang G-J, Logan J, Gatley SJ, Hitzemann R, Smith G, Fields F, Gur R, Wolf AP (1996) Dopamine transporters decrease with age in healthy subjects. J Nucl Med 37:554-558.

Volkow ND, Chang L, Wang G-J, Fowler JS, Leonido-Yee M, Franceschi D, Sedler M, Gatley SJ, Hitzemann R, Ding Y-S, Logan J, Wong C, Miller EN (2001) Dopamine transporter losses in methamphetamine abusers are associated with psychomotor impairment. Am J Psychiatry 158:377-382.

Wilson JM, Kish SJ (1996) The vesicular monoamine transporter, in contrast to the dopamine transporter, is not altered by chronic cocaine self-administration in the rat. J Neurosci 16:3507-3510.

Wilson JM, Kalasinsky KS, Levey AI, Bergeron C, Reiber G, Anthony RM, Schmunk GA, Shannak K, Haycock JW, Kish SJ (1996) Striatal dopamine nerve terminal markers in human, chronic methamphetamine users. Nat Med 2:699-703.

Woolverton WL, Ricaurte GA, Forno LS, Seiden LS (1989) Long-term effects of chronic methamphetamine administration in rhesus monkeys. Brain Res 486:73-78.

Zhang Y, Angulo JA (1996) Contrasting effects of repeated treatment vs. withdrawal of methamphetamine on tyrosine hydroxylase messenger RNA levels in the ventral tegmental area and substantia nigra zona compacta of the rat brain. Synapse 24:218-223. 